\section{Diagnostic de Babesia bigemina avec le test à l'immunoperoxydase}

\author{
T. Blandino ${ }^{1}$ \\ M. Barrera ${ }^{1}$ \\ M. Alonso ${ }^{1}$ \\ M. Faure ${ }^{1}$ \\ J.G. Rodriguez Diego ${ }^{1}$
}

BLANDiNO (T.), BARRERA (M.), ALONSO (M.), FAURE (M.), RODRIGUEZ DIEGO (J.G.). Diagnostic de Babesia bigemina avec le test à l'immunoperoxydase. Revue Elev. Méd. vét. Pays trop., 1995, 48 (3) : 244-246.

Une méthode de diagnostic sérologique de Babesia bigemina par l'immunoperoxydase a été mise au point; des lames d'antigène ont été réalisées à partir de sang parasité par $B$. bigemina et conservées à $-20^{\circ} \mathrm{C}$. Cent soixante-cinq sérums ont été testés en comparant le test a l'immunoperoxydase à l'immunofluorescence indirecte. Une coïncidence de 95 p. 100 a été observée entre les deux tests, ainsi qu'une spécificité relative de 95,5 p. 100 et une sensibilité relative de 94,8 p. 100 pour le test à l'immunoperoxydase, avec une valeur prédictive positive de 96,8 p. 100 Les résultats démontrent l'efficacité de ce test pour détecter les anticorps contre $B$. bigemina.

Mots-clés : Bovin - Babesia bigemina-Diagnostic - Cuba.

\section{Introduction}

A Cuba, la babésiose bovine due à Babesia bovis et à $B$. bigemina, est une des principales causes de mortalité chez les bovins adultes. Le diagnostic est réalisé par l'observation des parasites sur des frottis de sang. Cependant, lors d'infections chroniques la parasitémie est si basse que l'examen de frottis est peu efficace. Par contre, les tests sérologiques permettent de détecter les anticorps.

Le test d'immunofluorescence indirecte (IFI) est un test sérologique très sensible pour le diagnostic de B. bigemina (7) mais il pose des problèmes de spécificité et de subjectivité dans l'interprétation des résultats (3). Des tests ELISA sur lame $(2,4)$ ont été mis au point pour Babesia bovis, montrant une bonne efficacité et une facilité d'exécution. Le présent travail présente la mise au point du test à l'immunoperoxydase (IP) pour détecter les anticorps contre $B$. bigemina.

1. Laboratorio de Parasitología, Centro Nacional de Sanidad Agropecuaria, Apdo 10, San José de las Lajas, La Havane, Cuba.

Reçu le 19.4.1993, accepté le 8.11.1995.

\section{Matériel et Méthodes}

\section{Préparation de l'antigène}

La méthode a été décrite par Alonso et al. (1). Du sang infecté avec une parasitémie d'environ 4,3 p. 100 est lavé 4 fois avec du PBS. Les globules rouges sont dilués pour donner une dilution finale de 30 p. 100 de globules rouges dans du PBS avec 3,5 p. 100 d'alumine bovine.

Les lames sont frottées rapidement avec du PBS + 3,5 p. 100 d'albumine bovine pour faciliter l'étalement des globules rouges infectés. Les globules rouges sont étalés grâce à une centrifugeuse avec porte-lames à $2000 \mathrm{t} / \mathrm{min}$ pendant 20 secondes pour obtenir un étalement homogène. Les lames sont séchées 3 à 5 minutes à $56^{\circ} \mathrm{C}$, puis enveloppées et conservées à $-20^{\circ} \mathrm{C}$.

\section{Sérums}

Cent soixante-cinq sérums ont été comparés par le test à f'IP et par l'IFI :

- 27 témoins : 8 positifs vis-à-vis de $B$. bigemina, obtenus d'animaux infectés expérimentalement ; 8 négatifs provenant de veaux splénectomisés qui n'ont pas développé de parasitémie ; 11 séropositifs vis-à-vis d'autres hémoparasites (Theileria sp., Anaplasma sp., B. bovis);

- 138 provenant de fermes où la babésiose a ćtć dia gnostiquée sur des frottis.

\section{Congugués}

Pour le test IP, une immunoglobuline anti-IgG bovine obtenue sur lapin et conjuguée à la peroxydase a été utilisée à une dilution de $1: 500$, et pour l'IFI, une immunoglobuline anti-lgG bovine conjuguée à l'isothiocyanate de fluorescéine diluée à 1: 400 a été utilisée.

\section{Substrats}

Pour le test IP, les substrats étaient la 3-3 diaminobenzidine et le 4 chloro 1 naphtol.

\section{Réalisation du test IP}

Chaque lame d'antigène a été divisée en 12 compartiments avec un crayon marqueur et, dans chaque compartiment a été placé un papier filtre rond imprégné de sérum dilué. Les sérums témoins sont dilués au $1 / 100$, $1 / 200,1 / 400$ et $1 / 800$. Les sérums sont incubés à $37^{\circ} \mathrm{C}$ pendant $30 \mathrm{~min}$. Les lames sont lavées 3 fois avec du PBS et le conjugué ajouté en deux dilutions au 1/250 et $1 / 500$. Une nouvelle incubation à $37^{\circ} \mathrm{C}$ se déroule en 2 temps différents pour déterminer quel est le meilleur ( 30 
et $45 \mathrm{~min}$ ). Après un nouveau lavage avec le PBS, 2 substrats sont comparés (la diaminobenzidine et le 4 chloro 1 naphtol), avec une incubation de $30 \mathrm{~min}$ à $37^{\circ} \mathrm{C}$ et à température ambiante. La réaction est stoppée par un lavage à l'eau distillée, les lames montées avec de la glycérine tamponnée puis examinées avec un microscope optique équipé d'un objectif $100 \times$ à immersion.

\section{Test IFI}

C'est un test classique où les sérums sont dilués au $1 / 100$ et le conjugué au 1/400.

\section{Reproductibilité du test IP}

Le test a été répété 5 fois avec les sérums témoins.

\section{Sensibilité, spécificité et valeur prédictive}

Ces valeurs ont été calculées en comparant les deux tests et en utilisant la formule établie pour la validation des tests diagnostiques (6).

\section{Résultats}

Pour standardiser le test IP, les meilleurs résultats furent obtenus avec une dilution du sérum au 1/200 et un temps d'incubation de 30 minutes, et une dilution du conjugué au $1 / 500$ et un temps d'incubation de 45 minutes. Le substrat 4 chloro 1 naphtol a permis une meilleure différentiation des sérums positifs et négatifs après 30 minutes d'incubation à température ambiante.

La réaction positive était mise en évidence par une coloration bleu-violet des parasites et parfois de la membrane de l'érythrocyte qui contient un parasite, alors que rien n'est coloré dans la réaction négative.

Le test IP ne réagit ni avec $B$. bovis, ni avec Theileria sp., ni avec Anaplasma sp. (tab. I). Les 5 répétitions du test ont donné les mêmes résultats dans la détection des sérums témoins positifs et négatifs.

Pour les 165 sérums analysés avec les deux tests, 95 p. 100 des résultats coïncidèrent. Pour le test IP, une sensibilité relative de 94,8 p. 100 et une spécificité relative de 95,5 p. 100 furent calculées ainsi qu'une valeur prédictive positive de 96,8 p. 100 et une valeur prédictive négative de 97 p. 100 (tab. II).

\section{Discussion}

Le test IP apparaît spécifique de $B$. bigemina et donne des résultats comparables à ceux obtenus avec l'IFI sans nécessiter un microscope à immunofluorescence. Le
TABLEAU I

Résultats du test IP appliqué à des sérums témoins positifs et négatifs

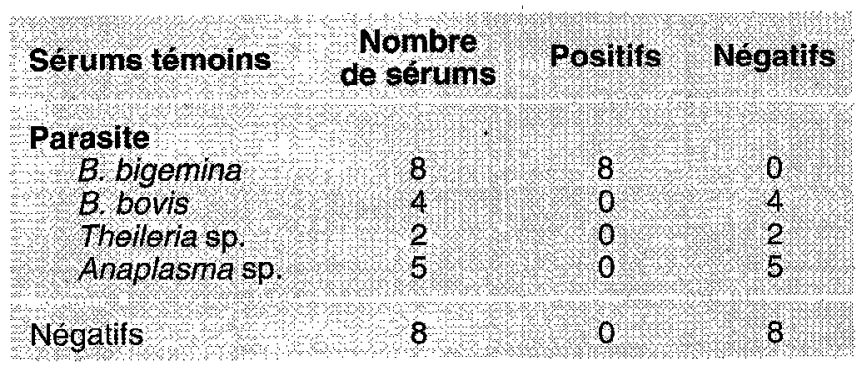

TABLEAU II

Comparaison des tests à l'immunoperoxydase el à immunofluorescence indirecte pour diagnostiquer Babesia bigemina

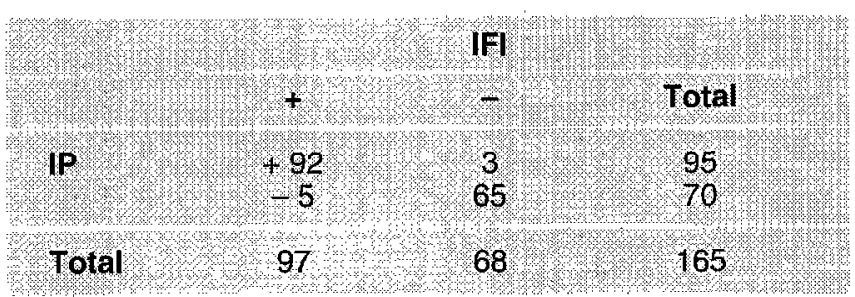

Sensibilité relative $=92 / 97=94,8$ p. 100 .

Spécificité relative $=65 / 68=95,6$ p. 100 .

Valeur prédictive positive $=92 / 95=96,8$ p. 100 .

Valeur prédictive négative $=65 / 70=92,9$ p. 100 .

tableau I présente une bonne reproductibilité de l'IP qui a démontré son efficacité dans la détection des anticorps anti- $B$. bigemina, confirmant les résultats obtenus avec les tests destinés à Plasmodium falciparum (5) et à $B$. bovis (2).

\section{Conclusion}

Le test IP peut être recommandé pour le diagnostic sérologique de $B$. bigemina, car il présente de bonnes sensibilité et spécificité, ainsi que l'avantage d'être facile à réaliser avec un simple microscope optique.

\section{Remerciements}

Nous remercions le Dr Emmanuel Camus, CIRAD-EMVT, Guadeloupe, pour sa traduction et ses corrections.

\section{Bibliographie}

1. ALONSO M., BLANDINO T., LARRAMENDI R., JIMENEZ T., MESA J., 1988. Immunofluorescencia indirecta en el diagnostico de la babesiosis hovina en Cuha. Revta Salud Anim., 10 : 197-203. 


\section{Communication}

2. BLANDINO T., BARRERA M., ALONSO M., 1991. Prueba de inmunoperoxidasa para el diagnostico de $B$. bovis. In : IV Congreso Internacional de Malaria y Babesiosis, Rio de Janeiro, Brasil, 1991.

3. GOFF W.L., PALMER G.H., MC ELWAIN T.F., DAVIS W.C., MC GUIRE T.C., 1989. Development of ELISA diagnostic test for Babesia infections, using highly immunogenic species specific and strain-common glycoproteins. In : Proc. 8th Natl Vet. Hemoparasites Disease Conference, St Louis, MO, USA, 1989, p. 353-376.

4. KUNG U.M.W., GOODGER B.V., 1990. A Slide Enzyme Linked Immunosorbent Assay (SELISA) for the diagnostic of Bebesia bovis infections and for the screening of Bebesia-specific monoclonal antibodies. Int. J. Parasitol, $20: 341-345$.

5. LIM T.S., 1988. A sensitive malaria immunoperoxidase assay for the detection of Plasmodium falciparum antibudy. Am. J. trop. Med. Hyg., 38 : $255-257$.

6. LOPEZ N.A., SCARLETT J.M., PALLOCK R.V.H., JACOBSON R.H., 1990. Sensitivity, specificity and predictive values of clinease virastat saliva test for feline leukemia virus infection. Cornell vet., 80 : 75-80.
7. ROSS J.P.J., LOHR K.F., 1967. Serological diagnosis of Babesia bigemina infection in cattle by the indirect fluorescent antibody test. Res. vet. Sci., 2 : $551-562$

BLANDINO (T.), BARRERA (M.), ALONSO (M.), FAURE (M.), RODRIGUEZ DIEGO (J.G.). Diagnosis of Babesia bigemina with the immunoperoxidase assay. Revue Élev. Méd. vét. Pays trop., 48 (3) : 244 246.

An immunoperoxidase assay for the serological diagnosis of Babesia bigemina was developed. The antigen slides were prepared from $B$. bigemina-infected blood and stored at $-20^{\circ} \mathrm{C}$. One hundred and sixly five sera were tested, comparing the immunoperoxidase assay to the indirect fluorescent antibody test. A coincidence of $95 \%$ was observed between both tests. For the immunoperoxidase assay, a relative sensitivity of $94.8 \%$, a relative specificity of $95.5 \%$, and a positive predictive value of $96.8 \%$ were calculated. The results demonstrated the efficacy of this technique for detecting antibodics to $B$. bigemina.

Key words : Cattle - Babesia bigemina - Diagnosis - Cuba. 